\title{
Biomechanics of Aligners: Literature Review
}

\author{
Sana Alami ${ }^{1}$, Amal EL Aouame ${ }^{1}$, Assma EL Moutawakil1* ${ }^{*}$, Fatima zahra Benammar ${ }^{2}$ and Farid EL Quars ${ }^{1}$ \\ ${ }^{1}$ Department of Orthodontics, Hassan II University, Morocco
}

${ }^{2}$ Private Practice, Morocco

Submission: December 23, 2020; Published: January 15, 2021

*Corresponding author: Assma EL Moutawakil, Abou Al Alaa Zahar street, 21100, Casablanca, Morocco

Keywords:

Introduction: This review aims to describe the aligners biomechanics, to discuss how tooth movement may be accomplished using these aligners, and the recent improvements and adjuncts incorporated.

Methods: The data collection for this literature review was conducted through: An electronic search of the articles through the databases: PUB MED - GOOGLE SCHOLAR - SCIENCE DIRECT, and a manual search. The research included human studies, clinical trials, in vitro studies, literature reviews, case reports, and books published between 2003 and 2020.

Results: At the end of the initial search, 2722 articles were preselected. 28 articles were included after full reading, containing clinical trials, in vitro studies, review articles, and books.

Discussion: In principle, aligners produce tooth movements through a motion-based system. The teeth move until they align with a position prefigured by the aligner. However, when root movement is required, aligners should be designed in such a way that specific forces are applied to the crowns, several studies indicate that composite attachments and power ridges have been very effective in these cases, in addition to the use of other auxiliaries that have improved the performance. Despite this progress, many authors still question the ability of aligners to treat complex malocclusions.

Conclusion: An in-depth study and a better understanding of aligners biomechanics are necessary so that clinicians can be able to suggest the appropriate treatment plans that best fit the needs of their patients, as well as a knowledge of the factors that influence the clinical mechanical behavior of aligners.

Keywords: Aligners; Biomechanics; Orthodontics

\section{Introduction}

Aligners treatment has been part of orthodontic practice for decades, especially since the introduction of Invisalign devices (Align Technology) in 1998. At first, aligners were used for mild to moderate orthodontic dental movements. Technological developments in aligner materials and production techniques, especially 3D technology to planning dental movements and the incorporation of attachments, allowed the improvement of the control of the position of the teeth in the three planes of space. This made possible the realization, by the aligners, of movements considered until then difficult or even impossible. However, it is recognized that many parameters influence the biomechanical characteristics of aligners such as material properties, material thickness, amount of activation, and use of attachments. Until 2003, few studies, adequately evaluated the biomechanics of aligners, have been published, leaving doubt among clinicians about the effectiveness of the appliance. Published articles mainly case reports. This literature review aimed to identify and review the orthodontic literature, and taking stock of the current state of knowledge regarding the biomechanics of aligners, to provide elements allowing a better understanding of the functioning of the technique of aligners, to improve clinical results and optimize dental movements.

\section{Methods}

\section{Search strategies}

Data collection for this literature review was carried out through:

i. An electronic search of the articles that are the subject of this literature review through the databases: PUB MED, GOOGLE SCHOLAR, SCIENCE DIRECT. 
On the various search engines, we have used the following keywords to identify the articles: clear aligner- orthodontic biomechanic - tooth movement.

For PUBMED, we used the keywords found in MeSH for English articles, according to the following search equations: Clear aligners AND orthodontic, Clear aligners AND biomechanic.

ii. A manual search to identify other references that could be included in the review. At the end of this research, we have selected 4 books.

We have respected the guidelines of the PRISMA charter in the treatment of references collected from the bibliographic research carried out.

\section{Eligibility criteria}

\section{a) Inclusion criteria}

i. Studies published during the last 17 years (2003-2020).

ii. Studies in humans.

iii. Literature reviews, Systematic reviews and metaanalysis responding to determined objectives.

iv. Studies of orthodontic treatment with aligners in adults.

v. Clinical tests. vi. Review and review articles.

vii. In vitro experimental studies.

viii. Case reports.

ix. Books.

\section{b) Exclusion criteria}

i. Animal studies.

ii. Studies published before 2003.

iii. Expert opinions.

\section{Results}

A preselection of articles was carried out by searching the keywords through the databases: During this step, 2795 articles were preselected. After applying the 2003-2020 Periodic Restriction filters + "humans" filter: we obtained 1192 articles. After reading headlines and eliminating duplicates, 140 articles were left. After reading the titles and abstracts, and considering the eligibility criteria, we kept 66 references. The full text of the articles was read to eliminate articles that did not meet the inclusion criteria. At the end of this step, the number of references obtained is 28 . Then we carried out a critical reading of the selected articles, during which we evaluated the validity of the articles to extract the necessary data (Figure 1).

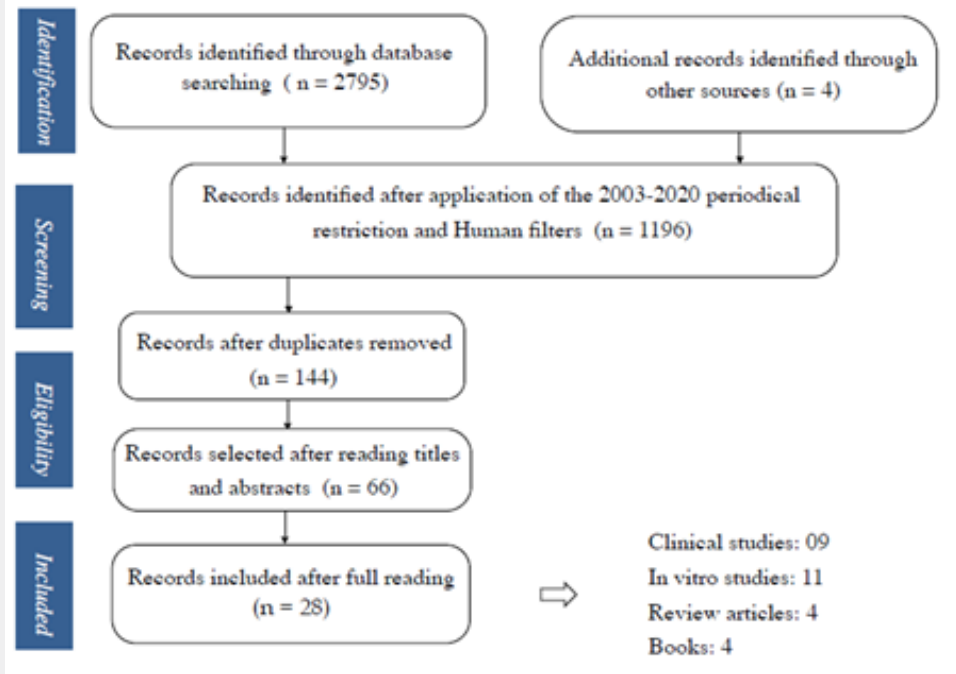

Figure 1: Search Strategy Diagram.

\section{Discussion}

\section{Force and displacement}

There are several types of forces that can be applied to the teeth: translation, torque, rotation, and extrusion require heavy forces of $50-150 \mathrm{~g}$, rocking motion requires $50-75 \mathrm{~g}$, intrusion requires light forces of $10-25 \mathrm{~g}$. Rocking motions require a single force, while translational movements require two or more force systems [1]. Barbagallo et al. [2] concluded that thermoplastic aligners have similar effects on root cement as light fixed orthodontic forces $(25 \mathrm{~g})$. This is explained by the discontinuous and removable nature of the force [2]. Castroflorio et al. [3] showed that Invisalign aligners produce an initial force of $1 \mathrm{~N}$ when distalizing a maxillary molar. To this end, aligners can induce the 
same biological response described for other fixed appliances, at least during the early stages of orthodontic treatment [3].

\section{Types of movements}

\section{The translational movement}

Translational movement is required in three main situations:

\section{a) Molar distalization}

A retrospective study was performed by Ravera et al. [4] the results of which showed that the translational movement of the maxillary molars was achievable with the use of aligners in combination with composite attachments and class II elastics [4]. The aligners allowed the maxillary second molars to be distalized by $2.25 \mathrm{~mm}$ without version movement or significant vertical crown movements. Another study by Simon et al. [5] in 2014, focused on evaluating the effectiveness of aligners regarding incisal torque movement, premolar derotation, and molar distalization. Molar distalization marked the highest rate of accuracy, approximately $87 \%$ without the use of Class II elastics during treatment [5] (Figures 2-4). .

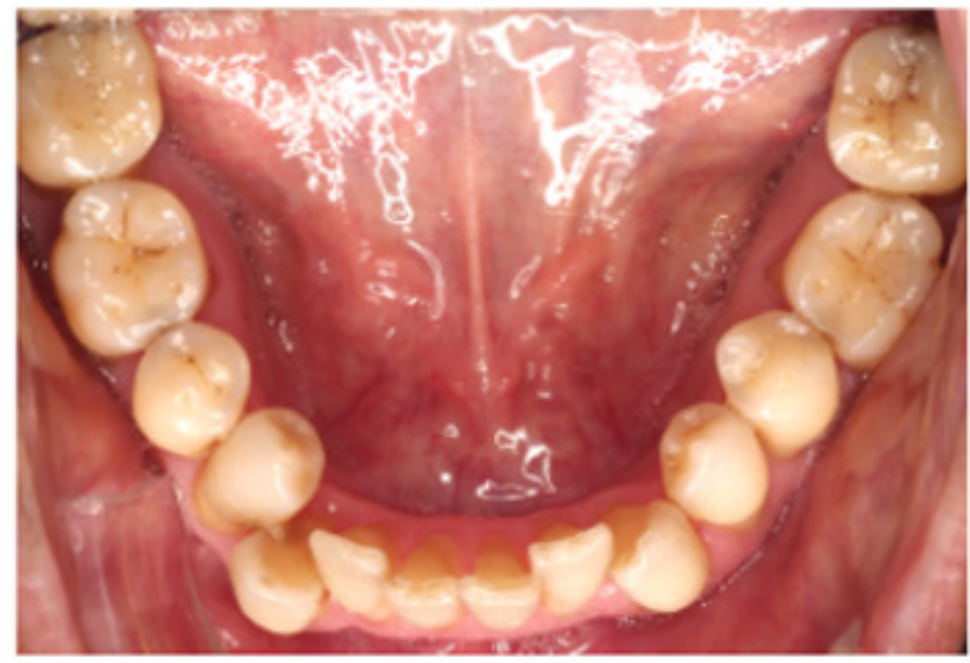

Figure 2: Occlusal view of the mandible before treatment.

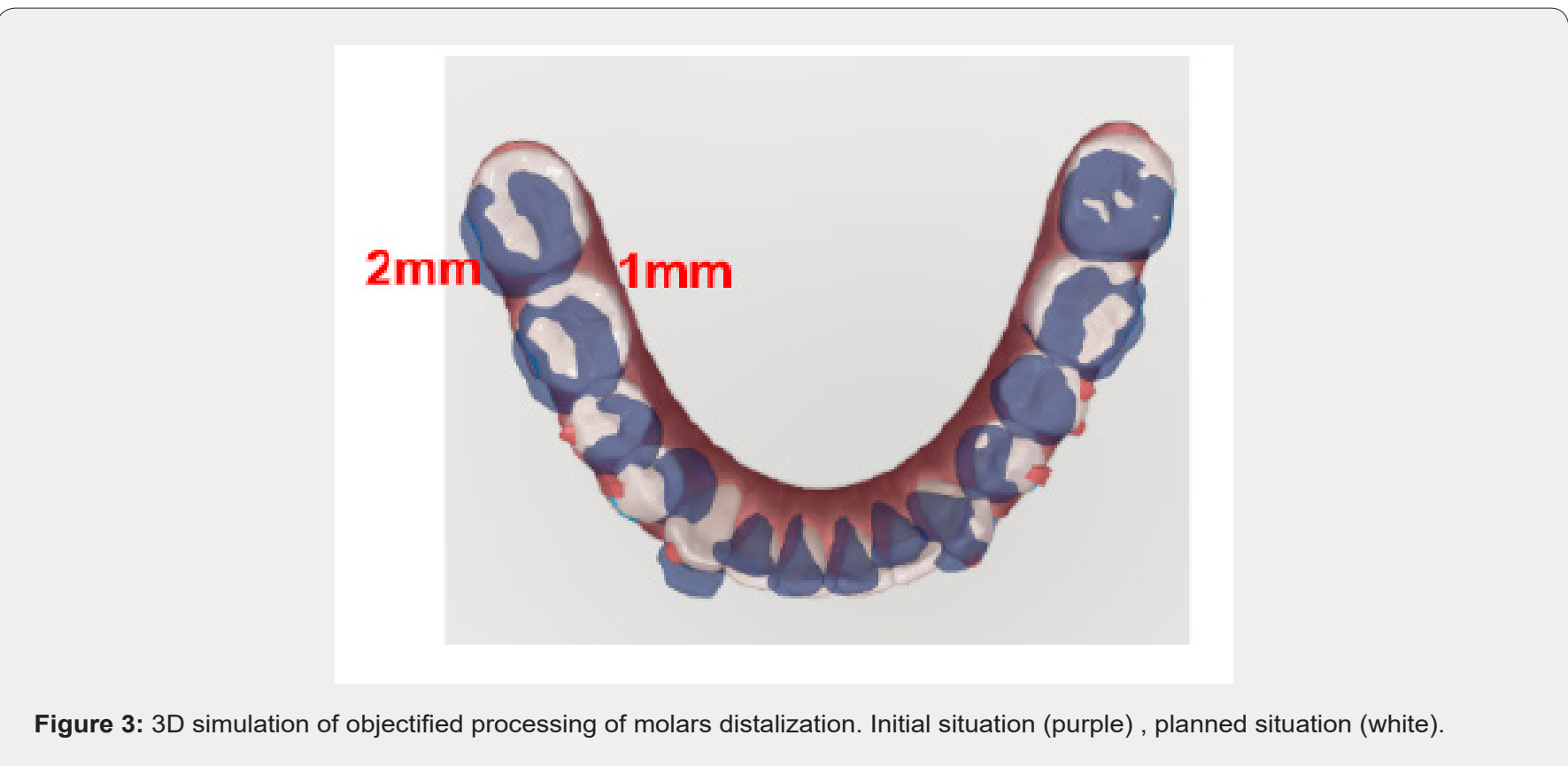




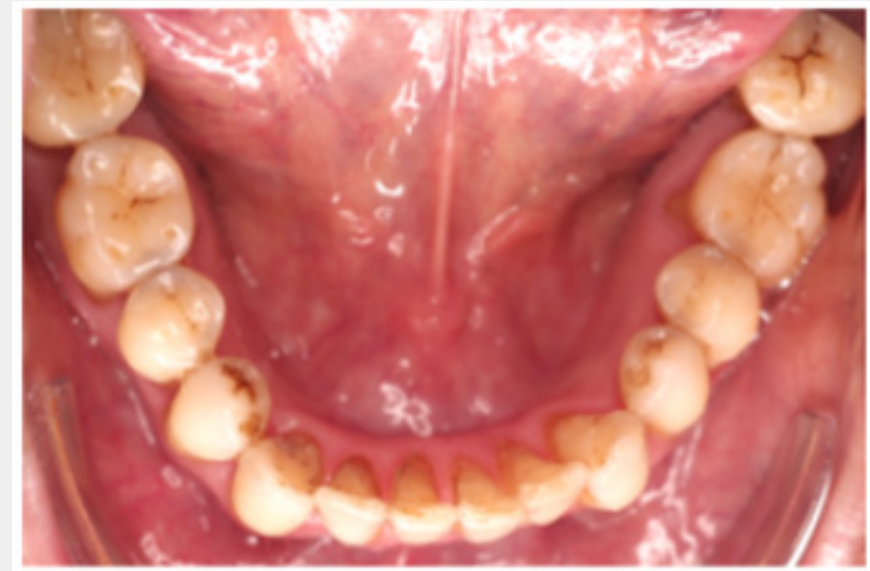

Figure 4: Occlusal view of the mandible - results of the initial phase of the aligner treatment: mandibular dental alignment resulting in distalization of the molars.

\section{b) Displacement of teeth adjacent to extraction sites}

Closure of space at the extraction sites requires "root control" attachments on the teeth adjacent to the extraction sites. The study by Li et al. [6] whose aim to evaluate the results of Invisalign treatment compared to the fixed multi-brace treatment [6] showed no significant difference between the two groups in the translation of the roots and the achievement of good root angulation. The results of this study could be due to the correct use of attachments to control the movement of the root [7]. This study confirmed that Invisalign can give good results in extraction cases (Figure 5).

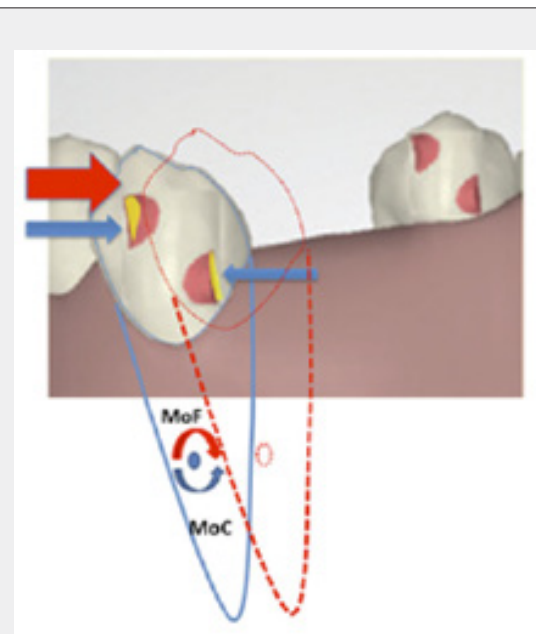

Figure 5: Schematic representation of the translational movement of a mandibular canine with ellipsoid attachments [7].

\section{c) Vestibulo-lingual / mesio-distal tooth displacement}

An in vitro study was performed by Elkholy et al. [8] in 2016 to study the forces and moments delivered by the new thinner PETG aligners during the labio-palatal translational movement of a maxillary central incisor led to the conclusion that for aligners without specific modifications such as pressure points specific or more rigid cervical regions to create a counter moment, the translational movement of the maxillary central incisors in the vestibular or palatal direction is impossible to achieve. According to Zhang et al. [9] the coronary movement produced ( $\sim 2.5 \mathrm{~mm})$ was relatively greater compared to the root movement $(\sim$ $0.4 \mathrm{~mm}$ ). These results indicate that the aligners cannot perform translational movements, which explains the lower quality of treatment and the high recurrence rate compared to the fixed multi-attachment treatment. According to the authors, the aligners moved the teeth mainly with version movements inducing an increase in stress in the cervical and apical regions. In a study by $\mathrm{Li}$ et al. [10], the orthodontic force in the lingual direction generated by the aligners increases as the alignment of the aligner increases. Except that the difference in the orthodontic forces produced between activation of $0.5 \mathrm{~mm}$ and $0.6 \mathrm{~mm}$ was not statistically 
significant. These results indicate that the activation of the aligner should not exceed $0.5 \mathrm{~mm}$ for the lingual translational movement of the maxillary central incisors. [10] In a study by Gomez et al. [11] the initial force system generated during translational movement of upper canines with aligners, with and without composite attachments was described [11].

\section{The extrusion movement}

The extrusion movement seems to be difficult to obtain. Indeed, in a prospective clinical study carried out by Kravitz et al. [12] the efficiency of tooth movement with removable polyurethane aligners was evaluated and the least accurate movement was the extrusion (29.6\%) - specifically, extrusion of the maxillary (18.3\%) and mandibular (24.5\%) central incisors. Also, an observational study by Tuncay et al. [13] examining tooth movement during upper lateral incisor eruption by aligners found that pure extrusion along the long axis is extremely difficult. This movement takes place in a non-linear movement for both the root and the crown, referred to as "wobbling". Kravitz et al.
[14] conduct a new study to provide an update on the accuracy of tooth movement with Invisalign. One of the most promising findings of this study was the improvement in the accuracy of maxillary incisor extrusion (55\%). That may be from the use of optimized extrusion attachments. It is then necessary to use attachments that have a so-called active flat surface, on which the aligner can exert force. The active part is the essential element of the attachment since it is the part that is engaged by the aligner. The rest of the attachment serves as a bond and a reinforcement [15]. These attachments should be beveled on the gum side. These attachments can be placed on a single tooth or group of teeth when multiple extrusions are required [16]. Beveled attachments are most often used when extruding a tooth. They can be 3 , 4, or $5 \mathrm{~mm}$ wide, $2 \mathrm{~mm}$ high, and 0.25 to $1.25 \mathrm{~mm}$ thick [7]. In open bite cases requiring absolute extrusion of the incisors, the optimized extrusion attachments are automatically placed on the incisors by the software when a pure extrusion of $0.5 \mathrm{~mm}$ or more is detected (Figures 6-8) [17].
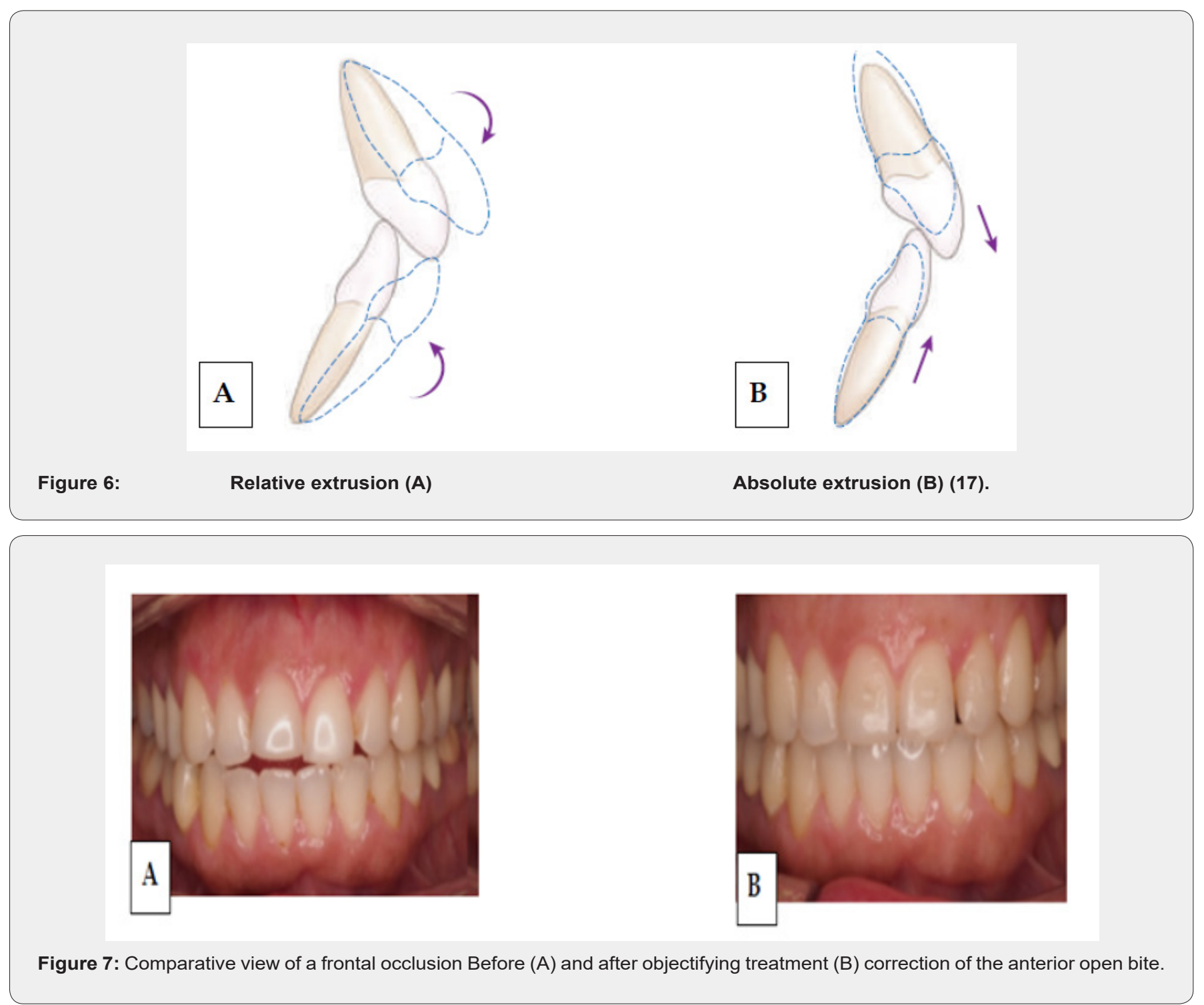


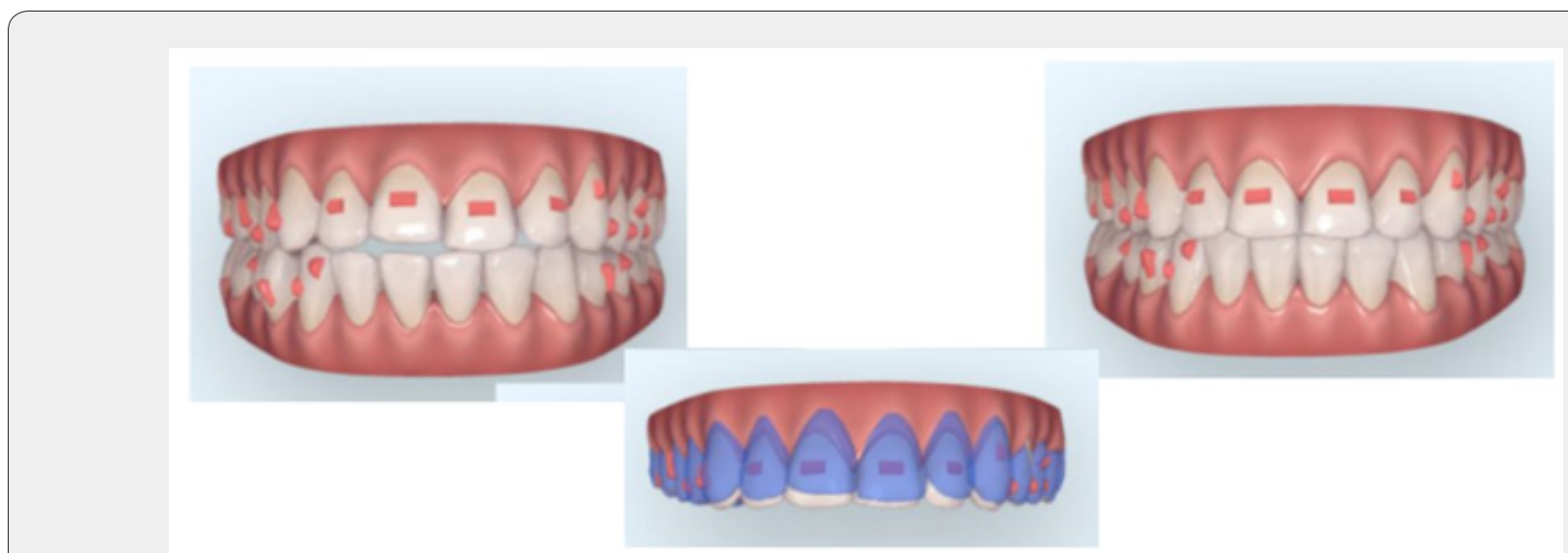

Figure 8: 3D simulation of the initial situation, objectified treatment, and dental overlays before and after treatment. The extrusion of the upper incisors requires attachements. It will be the result of absolute and relative extrusion.

Another method is to use a Power grip bonded to the buccal surface of the tooth affected by the extrusion, two buttons on the aligner at the adjacent teeth, and intra-jaw elastics. Extrusion is activated by changing the elastics 3-4 times a day. When the necessary extrusion is complete, the aligner locks the tooth in the desired position (Figure 9) [15]. In cases of open bite requiring 2 to $3 \mathrm{~mm}$ extrusions, intermaxillary elastics can be used. The extrusion teeth are provided with buttons and are attached to the opposing arch by rubber bands on the buttons as well. When the desired extrusion is complete, the target teeth will be in contact with the inner surface of the aligner and no further extrusion will occur (Figure 10). This method can be modified by placing the buttons on the lingual surface of the aligner in the same arch with the use of intra-arch elastics (Figure 11) [18].

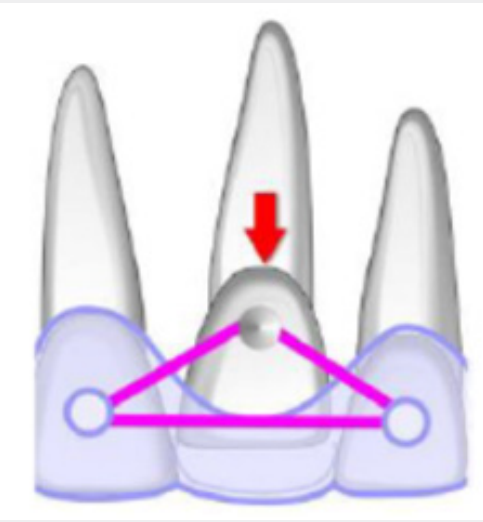

Figure 9: Power-Grip pressure relief device [22].

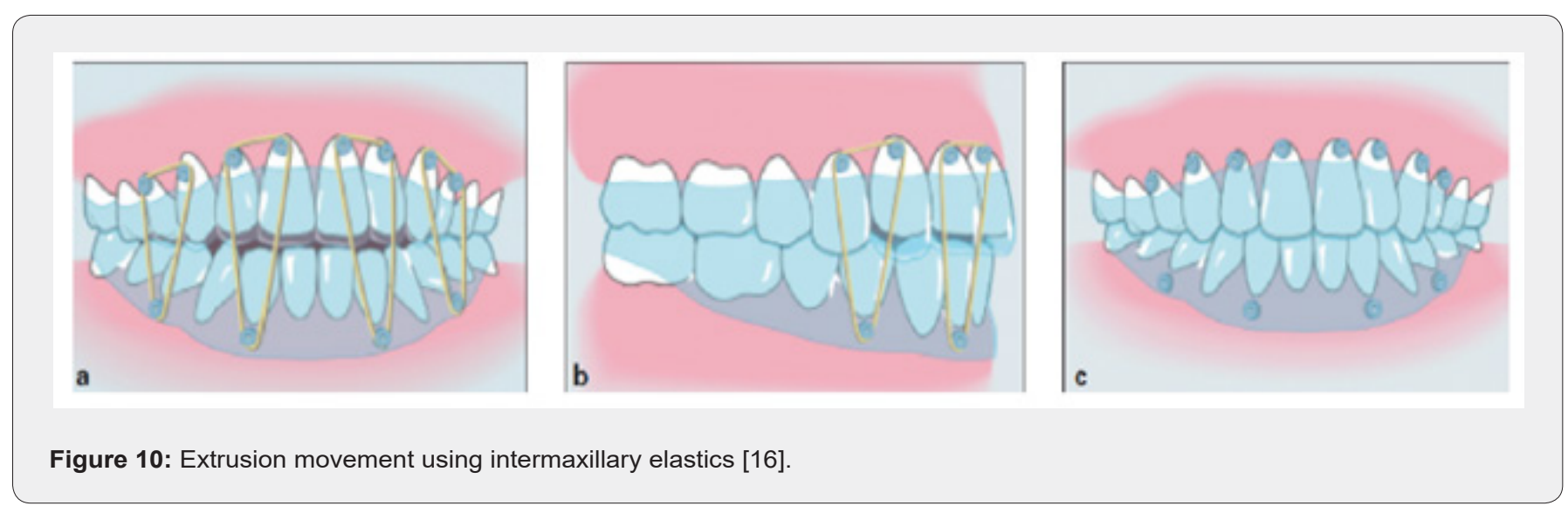



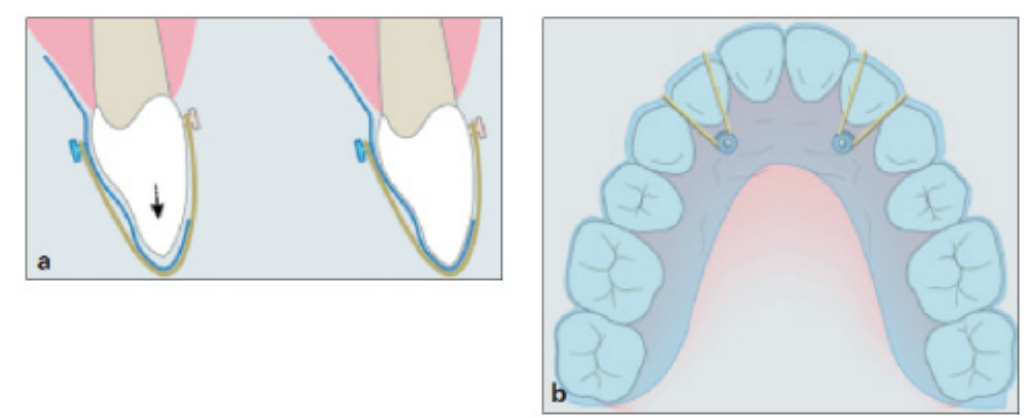

Figure 11: Sagittal (a) and occlusal (b) view showing the intra-arch elastic mooring procedure for incisor extrusion [16].

\section{The intrusion movement}

The best aligner treatment protocol to accomplish intrusion in a shorter period of time is to exert optimal intrusion force on the target teeth while maintaining stability of the anchorage teeth. The aligners have advantages because they cover all the teeth thus preventing the extrusion of the posterior teeth [19]. Intrusion normally does not require composite attachments on the affected teeth. However, the anchor teeth should be fitted with horizontal rectangular attachments for anchoring and relative extrusion of these premolars [20] (Figure 12). Another method is to position two buttons on the aligner buccally and lingually at the gum level of the target tooth. Two 1.5 to $2 \mathrm{~mm}$ deep grooves are then made on the incisal edge with a $1 \mathrm{~mm}$ diameter to hook the elastics in case of intrusion of the anterior tooth (Figure 13); in the case of a posterior tooth, the occlusal part of the aligner is cut [21].
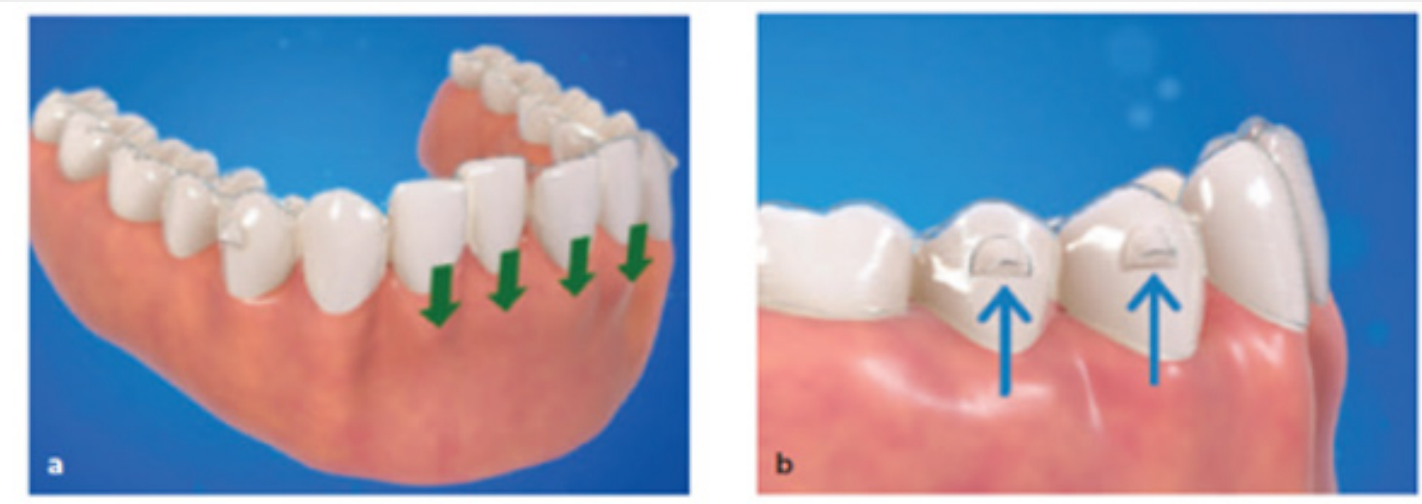

Figure 12: For optimized intrusion of the incisal sector, optimized passive attachments on the mandibular premolars provide anchorage preventing the removal of the aligner [21].
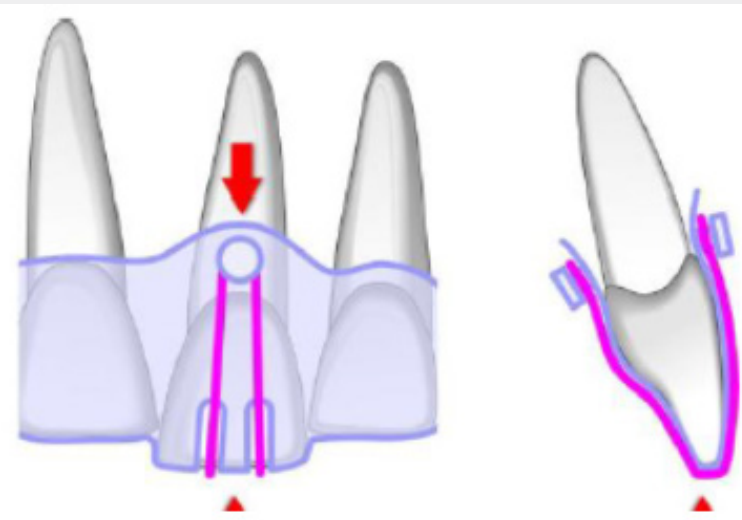

Figure 13: Intrusion of a central incisor using vestibular and lingual buttons to stretch an elastic generating the force of pressure [21]. 
The biomechanics of an ingressive force take into account the point of application of the force, but also the vestibulo-palatal / lingual inclination of the tooth. So, to intrude the teeth, it is necessary to correct the torque then the intrusion can be done in the cancellous bone by exerting a force on the tooth surface. Maintaining control of bucco-lingual tilt (torque) during intrusion is very important, because if the root apex is in contact with the buccal or lingual cortical bone, root resorption may occur [1]. A study was carried out by Saxena et al. [22] in 2010, showed an average tooth displacement rate of $0.9 \mathrm{~mm}$ per month for canines and $0.7 \mathrm{~mm}$ per month for incisors exposed to the same ingressive force. However, it has been observed clinically that the canines are more difficult to intrude than the incisors due to the length of the roots and their larger periodontal surface [22]. In an in vitro study by Yang Liu et al. [19] in 2018, the actual activation of the aligner on each tooth, the shape and position of the attachment, and the relative movement of adjacent teeth influenced the final intrusion force [19]. This study also showed that, for the same activation, the canines exhibit the greatest force of intrusion when they are intruded alone. To optimize the intrusive movement, the use of attachments on the affected teeth themselves may prove to be in favor of the

predictability of such movement.

\section{The tipping movement}

This is the easiest move to obtain when a force is applied against the crown.

\section{a) The uncontrolled tipping}

A study was carried out by Baldwin et al. [23] in 2006 to describe the movement of teeth adjacent to premolar extraction spaces during space closure with aligner appliances and then fixed appliances. In this study, treatment with aligners resulted in significant tipping of the teeth adjacent to premolar extraction sites [23].

\section{b) The controlled tipping}

As described in the literature, tipping is predictable with thermoplastic aligners, but it remains difficult to establish comparable root control [3,24]. However, there is a side effect of tooth movement with thermoplastic aligners called the 'water melon seed' effect, which refers to the unintentional intrusion of the displaced tooth triggered by an intrusive force caused by the distortion of the appliance [25]. A study was performed by Gao \& Wichelhaus [26] in 2017 to evaluate the effect of material thickness on the forces and moments delivered by aligners to a maxillary incisor during tipping and intrusion [26]. The calculated mean moment/force (M / F) ratio was between 8 and 9 (8.2) for tipping. This confirms the results of previous studies.

\section{The torque movement}

Torque movement with aligners can be done using Power Ridges. They are supposed to apply a lingual or palatal force on the cervical part of the crown, which, when constrained by the plastic covering the incisal edge of the same tooth, creates a couple of forces, this couple is supposed to produce the lingual torque. Another method used in the Essix system is also effective: using Hilliard thermoplier or one layer of composite, two forces are applied simultaneously buccally and lingually of the target tooth [21]. Controlling the torque of an upper central incisor requires the creation of effective couple of forces: [24,25] (Figures $14 \& 15$ ).
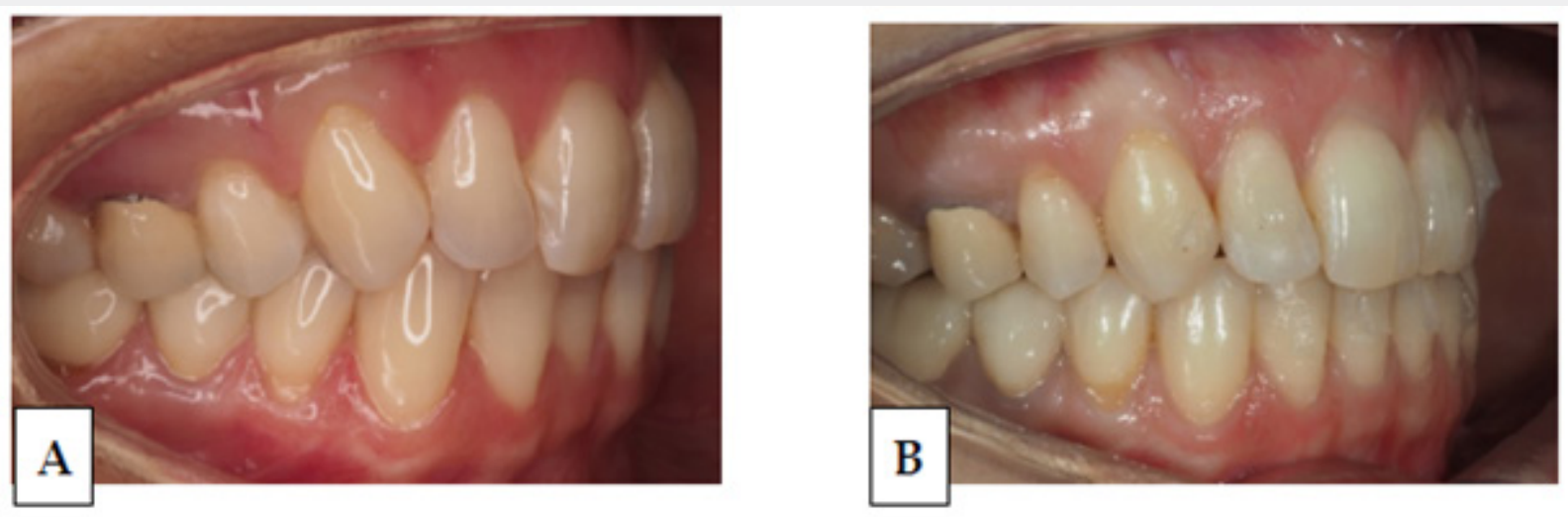

Figure 14: Comparative right lateral occlusion views before $(A)$ and after $(B)$ treatment showing the expression of torque in the upper incisors.

i. A tipping force, caused by a reversible deformation of the device near the gingival line

ii. A resultant force in the opposite direction, produced by the movement of the tooth against the opposing internal surface of the appliance near the incisal edge.
A study was carried out by Li et al. [6] in which they compare the results of the two treatments, one with an aligner and the other with fixed appliances. The results showed no significant difference between the two groups [6]. Although, the results showed that Invisalign might not produce enough radicular torque, especially in the posterior region. 


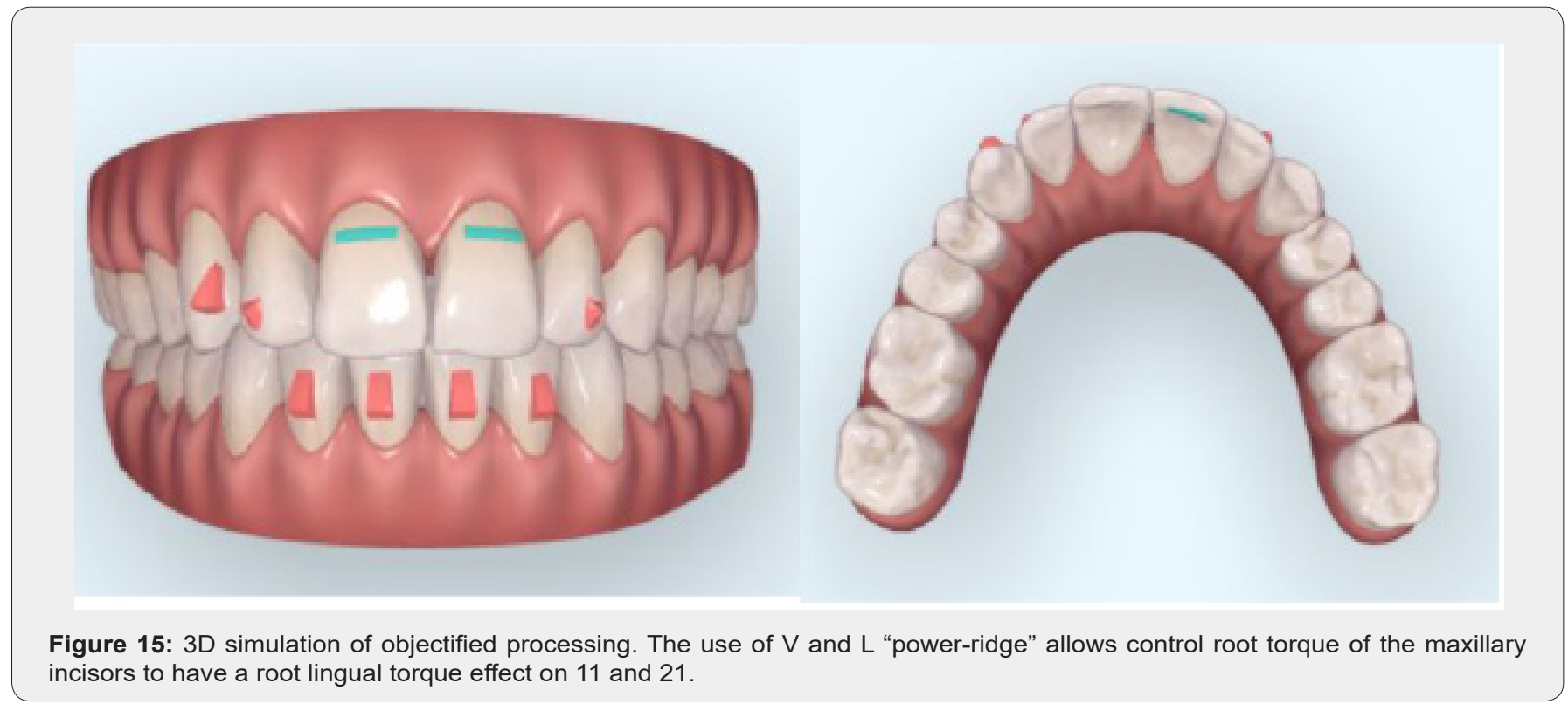

\section{The rotation movement}

Hahn et al. [27] found that only a slight activation of $\pm 0.17 \mathrm{~mm}$ or $0.5^{\circ}$ per step during rotation could produce ideal forces estimated between 0.35 and 0.6 N. 22 [27].

There are 2 methods to perform the rotation:

i. A couple of forces can be created to rotate a tooth by bonding buttons to the buccal and palatal surface of the rotated tooth and use elastic chains between them before or during the aligner treatment. The chains can be changed every 3 weeks until the rotation is corrected [28].

ii. It is also possible to perform canine and bicuspid rotations in a more predictable way using optimized rotation attachements.

These attachments have different shapes depending on the direction of force required to rotate the affected tooth (Figures 16 \& 17).

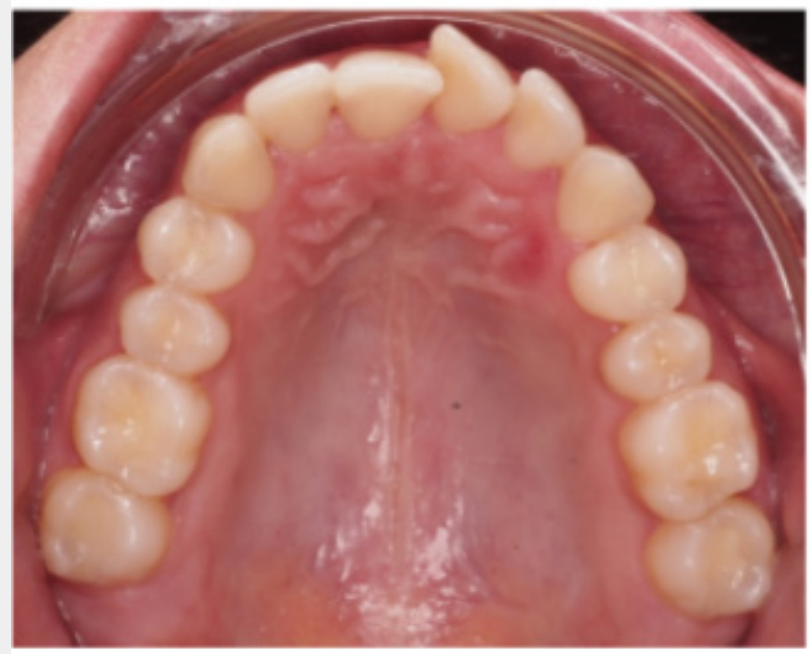

Figure 16: Occlusal view of the maxilla before treatment objectifying the rotation of 21,22 and 23.

The rotation of the 21, 22 was performed by the aligner himself, while an optimized attachment positioned distal of the 23 allowed for correction of the mesio-palatal rotation.
A study by Hahn et al. [27] in 2010, to evaluate the forces and moments exerted by removable thermo-plastic appliances produced by three different, hard thermoplastic materials, with 
the same thickness, on a maxillary central incisor during rotation in vitro, reported that it is also possible to measure an intrusive force, a general phenomenon associated with thermoplastic appliances called the "watermelon seed effect [27]. These intrusive forces can be explained by local deformation of the inclined inner surfaces of the appliance and with a reduced fit during progressive axial rotation, which tends to deform the appliance like a bow, by raising it in the anterior region [27]. Hahn et al. [28], Mencattelli et al. [29], Elkholy et al. [30] made the same observation. So, there is no pure rotation, but intrusion and tipping also occur. A general phenomenon was also observed, the forces and moments for anticlockwise activation were larger than for clockwise rotation.

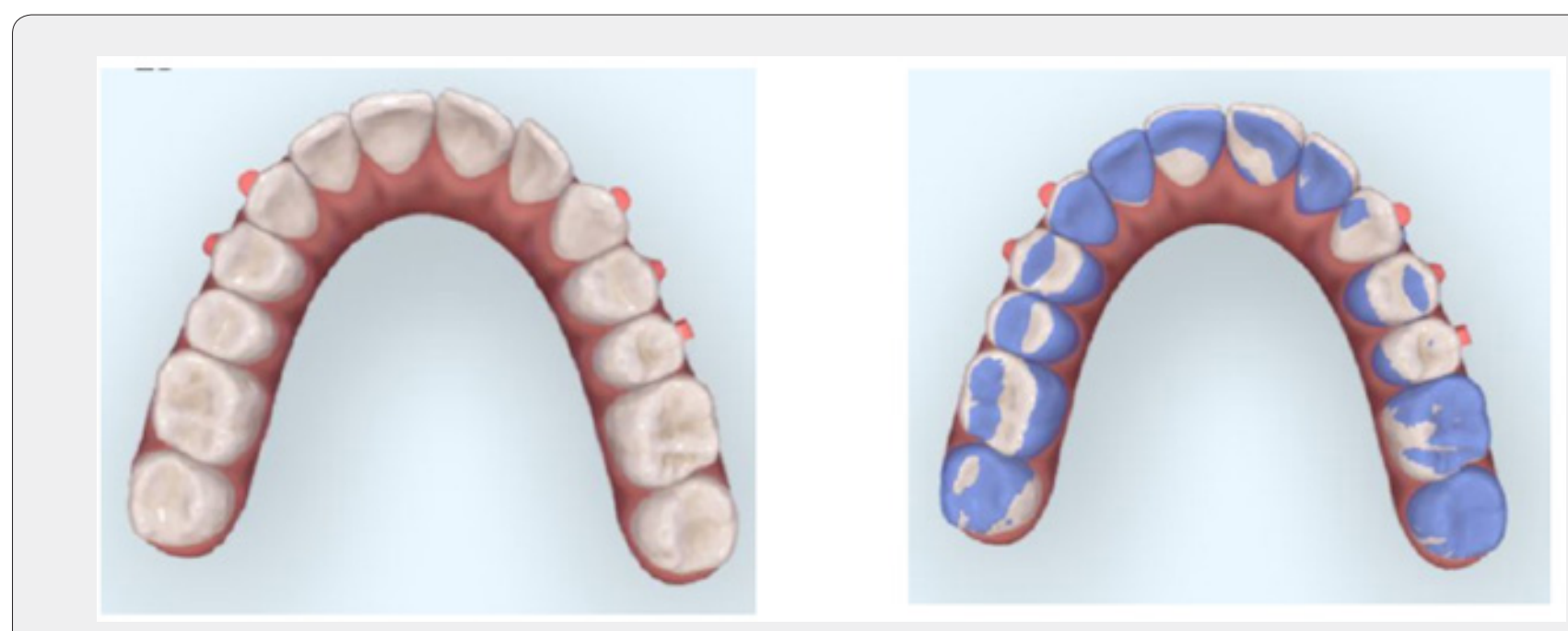

Figure 17: 3D simulation of objectified processing and dental superimpositions before and after treatment.

\section{Conclusion}

Orthodontic tooth movement is the result of the complex interaction of forces generated during the simultaneous movement of several dental units and biological events (apposition and resorption) that cannot be simulated in vitro, in addition to the complex chemical composition of the oral environment. In fact, additional studies are therefore needed to explore the biomechanical behavior of the aligner in vivo, allowing a better understanding of clinical performance within different aligner treatment systems.

\section{References}

1. Nguyen TQ (2014) The efficacy of clear aligners in tooth movement. Master of Science in Specialized Orthodontics. International Medical College.

2. Barbagallo LJ, Jones AS, Petocz P, Darendeliler MA (2008) Physical properties of root cementum: Part 10. Comparison of the effects of invisible removable thermoplastic appliances with light and heavy orthodontic forces on premolar cementum. A microcomputedtomography study. American Journal of Orthodontics and Dentofacial Orthopedics 133(2): 218-227.

3. Castroflorio T, Gamerro EF, Caviglia GP, Deregibus A (2017) Biochemical markers of bone metabolism during early orthodontic tooth movement with aligners. The Angle Orthodontist 87(1): 74-81.

4. Ravera S, Castroflorio T, Garino F, Daher S, Cugliari G, et al. (2016) Maxillary molar distalization with aligners in adult patients: a multicenter retrospective study. Progress in Orthodontics 17(1): 12.

5. Simon M, Keilig L, Schwarze J, Jung BA, Bourauel C (2014) Treatment outcome and efficacy of an aligner technique-regarding incisor torque, premolar derotation and molar distalization. BMC Oral Health 14(1): 68.

6. Li W, Wang S, Zhang Y (2015) The effectiveness of the Invisalign appliance in extraction cases using the $\mathrm{ABO}$ model grading system: a multicenter randomized controlled trial. International Journal of Clinical and Experimental Medicine 8(5): 8276.

7. Hennessy J, Al-Awadhi EA (2016) Clear aligners generations and orthodontic tooth movement. Journal of Orthodontics 43(1): 68-76.

8. Elkholy F, Schmidt F, Jäger R, Lapatki BG (2016) Forces and moments delivered by novel, thinner PET-G aligners during labiopalatal bodily movement of a maxillary central incisor: an in vitro study. The Angle Orthodontist 86(6): 883-890.

9. Zhang XJ, He L, Guo HM, Tian J, Bai YX, et al. (2015) Integrated threedimensional digital assessment of accuracy of anterior tooth movement using clear aligners. The Korean Journal of Orthodontics 45(6): 275281.

10. Li X, Ren C, Wang Z, Zhao P, Wang H, et al. (2016) Changes in force associated with the amount of aligner activation and lingual bodily movement of the maxillary central incisor. The Korean Journal of Orthodontics 46(2): 65-72.

11. Gomez JP, Peña FM, Martínez V, Giraldo DC, Cardona CI (2015) Initial force systems during bodily tooth movement with plastic aligners and composite attachments: A three-dimensional finite element analysis. The Angle Orthodontist 85(3): 454-460.

12. Kravitz ND, Kusnoto B, BeGole E, Obrez A, Agran B (2009) How well does Invisalign work? A prospective clinical study evaluating the efficacy of tooth movement with Invisalign. American Journal of Orthodontics and Dentofacial Orthopedics 135(1): 27-35. 
13. Tuncay OC, Keenan EJ (2017) Observational study of movements in lateral extrusion. In Seminars in Orthodontics 23(1): 103-106.

14. Haouili N, Kravit ND, Vaid NR, Ferguson DJ, Makki L (2020) Has Invisalign improved? A prospective follow up study on the efficacy of tooth movement with Invisalign. American Journal of Orthodontics and Dentofacial Orthopedics 158(3): 420-425.

15. Schupp W, Haubrich J (2017) Les aligneurs en orthodontie diagnostic, biomécanique, planification et traitements. Quintessence Int. ( $1^{\text {st }}$ edn), p. 358.

16. Glaser BJ (2017) Insider's guide to Invisalign treatment: a step-by-step guide to assist you with your Clin Check treatment plans. Sacramento. CA, p. 319.

17. Tai S (2018) Clear aligner technique. ( $\left(1^{\text {st }}\right.$ edn), Quintessence Publishing Co Inc, US, p. 309.

18. Park JH, Kim TW (2009) Open-bite treatment utilizing clear removable appliances with intermaxillary and intramaxillary elastics. World Journal of Orthodontics 10(2): 130-132.

19. Liu Y, Hu W (2018) Force changes associated with different intrusion strategies for deep-bite correction by clear aligners. The Angle Orthodontist 88(6): 771-778.

20. El-Bialy T, Galante D, Daher S (2016) Orthodontic Biomechanics: Treatment of Complex Cases Using Clear Aligner. Bentham Science Publishers 39(3): 342.

21. Chaudret F (2018) Biomécanique des aligneurs en orthodontie. Sciences du Vivant [q-bio]. dumas-01938617.

22. Saxena R, Kumar PS, Upadhyay M, Naik V (2010) A clinical evaluation of orthodontic mini-implants as intraoral anchorage for the intrusion of maxillary anterior teeth. World Journal of Orthodontics 11(4): 346351.
23. Baldwin DK, King G, Ramsay DS, Huang G, Bollen AM (2008) Activation time and material stiffness of sequential removable orthodontic appliances. Part 3: premolar extraction patients. American journal of orthodontics and dentofacial orthopedics 133(6): 837-845.

24. Smith RJ, Burstone CJ (1984) Mechanics of tooth movement. American Journal of Orthodontics 85(4): 294-307.

25. Brezniak N (2008) The Clear Plastic Appliance. A Biomechanical Point of View. The Angle Orthodontist 78(2): 381-382.

26. Gao L, Wichelhaus A (2017) Forces and moments delivered by the PET-G aligner to a maxillary central incisor for palatal tipping and intrusion. The Angle Orthodontist 87(4): 534-541.

27. Hahn W, Engelke B, Jung K, Dathe H, Fialka-Fricke J, et al. (2010) Initial forces and moments delivered by removable thermoplastic appliances during rotation of an upper central incisor. The Angle Orthodontist 80(2): 239-246.

28. Hahn W, Engelke B, Jung K, Dathe H, Kramer, et al. (2011) The influence of occlusal forces on force delivery properties of aligners during rotation of an upper central incisor. The Angle Orthodontist 81(6): 1057-1063.

29. Mencattelli M, Donati E, Cultrone, M, Stefanini C (2015) Novel universal system for 3-dimensional orthodontic force moment measurements and its clinical use. American Journal of Orthodontics and Dentofacial Orthopedics 148(1): 174-183.

30. Elkholy F, Mikhaiel B, Schmidt F, Lapatki BG (2017) Mechanical load exerted by PET-G aligners during mesial and distal derotation of a mandibular canine. Journal of Orofacial Orthopedics/Fortschritte der Kieferorthopädie 78(5): 361-370.

\begin{tabular}{|l|}
\hline \multicolumn{1}{|c|}{ Your next submission with Juniper Publishers } \\
will reach you the below assets \\
- Quality Editorial service \\
- Swift Peer Review \\
- Reprints availability \\
- E-prints Service \\
- Manuscript Podcast for convenient understanding \\
- Global attainment for your research \\
- Manuscript accessibility in different formats \\
( Pdf, E-pub, Full Text, Audio) \\
- Unceasing customer service \\
Track the below URL for one-step submission \\
https://juniperpublishers.com/online-submission.php \\
\hline
\end{tabular}

